Rev. Int. Contam. Ambie. 35 (1) 101-114, 2019

DOI: 10.20937/RICA.2019.35.01.07

\title{
EVALUACIÓN DEL RIESGO AMBIENTAL DE METALES PESADOS EN LOS SEDIMENTOS SUPERFICIALES DEL SACO DEL GOLFO DE CARIACO
}

\author{
María Valentina FUENTES-HERNÁNDEZ ${ }^{1 *}$, Octavio Antonio SANGUINETTI-GAMBOA² \\ y Luisa Lucina ROJAS DE ASTUDILLO ${ }^{1}$
}

${ }^{1}$ Universidad de Oriente (UDO), Núcleo de Sucre, Escuela de Ciencias, Avenida Universidad, Cerro Colorado, Cumaná 6101, Estado Sucre, Venezuela

${ }^{2}$ Universidad Politécnica Antonio José de Sucre (UNEXPO), Carrera Guri, Ciudad Guayana 8050, Estado Bolívar, Venezuela

*Autora para correspondencia: mariavalentinaf_1@hotmail.com

(Recibido septiembre 2017; aceptado mayo 2018)

Palabras clave: factor de enriquecimiento, índice de geoacumulación, probabilidad tóxica

\section{RESUMEN}

El objetivo de esta investigación fue evaluar la potencialidad de algunos metales pesados para provocar daños al ecosistema del golfo de Cariaco. Para ello se determinaron las concentraciones de los metales en los sedimentos y los índices de contaminación y riesgo ambiental. Además, se realizó una prueba de supervivencia con Artemia salina. Las concentraciones totales ( $\mu \mathrm{g} / \mathrm{g})$ fueron: $\mathrm{Cu}(29-91, \bar{X}=58), \mathrm{Cd}(1.9-4.9, \bar{X}=3.7)$, $\mathrm{Cr}(4-36, \bar{X}=21), \mathrm{Ni}(10-33, \bar{X}=21), \mathrm{Pb}(3-9, \bar{X}=6)$ y Zn $(32-112, \bar{X}=75)$. Todas las concentraciones de metales fueron menores que los estándares de calidad de sedimentos marinos (SQS). En la fracción biodisponible, los metales mostraron concentraciones no tóxicas. El factor de enriquecimiento $(\mathrm{FE})$ reveló incrementos por fuentes antrópicas en $\mathrm{Zn}, \mathrm{Cd}$ y Cu. El índice de geoacumulación (Igeo) indicó contaminación por $\mathrm{Cd}$. El código de riesgo ambiental (CRA) mostró que las concentraciones de $\mathrm{Cd}$ y $\mathrm{Pb}$ fueron inocuas; las de $\mathrm{Cr}, \mathrm{Cu}$ y $\mathrm{Ni}$ entre inocuas y poco peligrosas, y las de $\mathrm{Zn}$ y $\mathrm{Mn}$ medianamente peligrosas. Finalmente, las concentraciones de $\mathrm{Cd}$, $\mathrm{Cu}$ y Ni se ubicaron entre el intervalo de efectos bajos (ERL) y medios (ERM), y la potencialidad tóxica combinada (m-ERM-Q) fue baja (21\%). A pesar de esto, ningún ejemplar de Artemia salina tuvo efecto negativo en los extractos sedimentarios. Por tanto, el ecosistema se considera saludable, condición favorecida por sedimentos muy finos y anóxicos que benefician la retención de metales.

Key words: enrichment factor, geoaccumulation index, toxic probability

\begin{abstract}
The aim of this study was to assess the potential risk of heavy metals to the ecosystem of the gulf of Cariaco. To this end, we determined the concentration of heavy metals and the contamination and environmental risk indices in sediments. In addition, a survival test with Artemia salina was applied. The total metal concentrations $(\mu \mathrm{g} / \mathrm{g})$ were: $\mathrm{Cu}$ (29-91, $\bar{X}=58), \mathrm{Cd}(1.9-4.9, \bar{X}=3.7), \mathrm{Cr}(4-36, \bar{X}=21), \mathrm{Ni}(10-33, \bar{X}=21), \mathrm{Pb}(3-9$, $\bar{X}=6)$ and $\operatorname{Zn}(32-112, \bar{X}=75)$. Metal concentrations were lower than the sediment
\end{abstract}


quality standards (SQS). In the bioavailable fraction of sediments, the concentration of metals was innocuous. The enrichment factor (EF) showed that $\mathrm{Zn}, \mathrm{Cd}$ and $\mathrm{Cu}$ were enriched by anthropogenic sources. The geoaccumulation index (Igeo) revealed high contamination by $\mathrm{Cd}$. The risk assessment code (RAC) showed that the concentrations of $\mathrm{Cd}$ and $\mathrm{Cr}$ were innocuous, $\mathrm{Cu}$ and $\mathrm{Ni}$ were between harmless and scarecely dangerous, and $\mathrm{Zn}$ and $\mathrm{Mn}$ were moderately hazardous. Finally, $\mathrm{Cd}, \mathrm{Cu}$ and $\mathrm{Ni}$ were the only metals with concentrations ranging between effects range low (ERL) and median (ERM) and the combined toxic potential of the metals was low (21\%). Despite this, no specimen of Artemia salina had negative effects on sedimentary extracts. Therefore, the ecosystem can be considered healthy, which is favored by very fine and anoxic sediments that lead to accumulation of metals.

\section{INTRODUCCIÓN}

El golfo de Cariaco es una depresión ubicada en la zona nororiental de Venezuela, al este de la fosa de Cariaco, que separa la región continental de la península de Araya en el estado Sucre. Su gran importancia económica, ecológica y ambiental radica en la diversidad de ecosistemas acuáticos tropicales y en su elevada productividad primaria ocurrente en dos periodos anuales de surgencia, la cual favorece la presencia de abundantes poblaciones de plancton, necton y bentos (Lodeiros 2002, Núñez et al. 2011). Particularmente, su extremo más oriental es un área resguardada geomorfológicamente, bordeada de tupidos manglares, considerada como uno de los ecosistemas más productivos del mundo, con presencia de praderas de Thalassia testudinum y aguas muy tranquilas y fértiles. Estas características condicionan al área como un vivero o criadero natural, zona de desove de especies marinas, resguardo de fauna silvestre y protección de la línea de costa (Fuentes et al. 2010, Nowrousi et al. 2014). Durante la época de lluvias, cuyos máximos ocurren entre octubre y noviembre, las escorrentías estacionales continentales y el río Cariaco o Carinicuao transportan detritos rocosos y material vegetal, aportando energía, alimentos, vitaminas y minerales para la biota. Además, los procesos de afloramiento y renovación de agua estimulan la producción fitoplanctónica que junto a restos de manglares y otras partículas sedimentan y enriquecen el fondo marino (Márquez et al. 2011), de tal manera que las texturas sedimentarias varían desde arenosa media fina hasta limo arcillosa (Quintero et al. 2006).

El saco del golfo de Cariaco colinda con los municipios Mejías y Ribero. E1 51.4 \% de su población actual (74 810 habitantes), estimada en el último censo (INE 2014), radica en sus riberas y depende de los recursos que allí se generan. En ambas costas, el servicio de cloacas, prácticamente, no existe: $95.7 \%$ de la costa norte y $81.4 \%$ de la costa sur carecen de este (Quintero et al. 2002). De hecho, los habitantes descargan especies químicas potencialmente nocivas directamente al golfo con las aguas servidas y desechos sólidos. Muchos de sus pobladores están dedicados a la pesca artesanal y usan motores que requieren combustibles fósiles (diesel, gasolina y aceite) y otros practican actividades agrícolas (26 932 habitantes) que incrementan los aportes antrópicos. Además, recibe contribuciones del río Cariaco, criaderos de camarones, escorrentías límnicas y tráfico automotor (Márquez et al. 2005). Todas estas descargas son distribuidas por el sistema de corriente reinante desde el litoral sur hasta el norte (PNUMA 2009, Montezuma 2010).

Aunque en los ambientes marino-costeros, los metales pesados suelen estar presentes en concentraciones bajas, éstas pueden aumentar por las entradas terrígenas y los aportes antrópicos, más que por el enriquecimiento natural del sedimento, derivado de la meteorización geológica (Ntakirutimana et al. 2013). De hecho, los sedimentos median la captación, el almacenamiento, la liberación y la transferencia de los metales entre los compartimientos ambientales. Por lo tanto, este acrecentamiento causa problemas ambientales, como el deterioro del ecosistema y de los propios recursos vivos, además de comprometer la seguridad alimentaria y la salud pública (Reyes et al. 2016).

Los metales pesados presentan una elevada capacidad para adsorberse en material particulado, y son depositados en los sedimentos cuando disminuye la energía del medio que los transporta. Además, pueden coprecipitar o formar complejos sobre la superficie de las partículas y revestimientos. Esto afecta negativamente a las comunidades residentes, usualmente comunidades bénticas, relativamente sésiles, en contacto directo con sedimentos contaminados. La adsorción de los metales es un proceso competitivo entre los diferentes iones presentes por los centros 
activos del sedimento y, por tanto, está fuertemente influenciada por la naturaleza de la matriz mineral, el tamaño de las partículas sedimentarias, las características físicas y químicas del medio, el $\mathrm{pH}$, los procesos de difusión y mezcla, la actividad de los organismos bentónicos y la resuspensión (Ponce et al. 2000, Calderón y Valdés 2012, Nowrousi et al. 2014). De hecho los sedimentos son reservorios de metales pesados que pueden actuar como una fuente no puntual, liberar metales y otros contaminantes a las aguas superpuestas por procesos naturales o antrópicos, y provocar efectos adversos (Wang et al. 2010, El Nemr et al. 2012).

Debido a la gran importancia de los sedimentos en el ciclo global ecológico de metales pesados $(\mathrm{Cd}$, $\mathrm{Cr}, \mathrm{Cu}, \mathrm{Ni}, \mathrm{Pb}, \mathrm{Zn}$ ) en los compartimientos marinos y la posibilidad de provocar daños ecológicos y biológicos, el principal objetivo de este estudio es evaluar si existe una amenaza concreta de daño por metales pesados en los sedimentos del saco del golfo de Cariaco y sus posibles efectos en las condiciones ambientales. Esto es imperativo para asegurar su sostenibilidad futura, dado que los metales pesados son persistentes, no biodegradables, variablemente tóxicos, acumulativos, fácilmente asimilables por el fitoplancton y por los organismos filtradores, y son transferidos en la cadena alimenticia.

Para evaluar el riesgo de metales pesados en el sedimento se ha utilizado una variedad de métodos frecuentemente aplicados como el factor de enriquecimiento (FE) de sedimentos, el índice de acumulación geológica (Igeo), las directrices de calidad de sedimentos, el cociente promedio de los valores del intervalo de efectos medianos (m-ERM-Q), el código de evaluación de riesgo ambiental (CRA) y la supervivencia de Artemia salina (Serveiss y Ohlson 2007, Sung et al. 2010, Bangqi et al. 2013). Los resultados de esta evaluación brindarán a los responsables de la formulación de políticas, a los gestores de recursos y al público, métodos sistemáticos que guiarán la toma de decisiones para proteger dicho sedimento y mejorar los procedimientos de protección contra un posible riesgo mayor (Ntakirutimana et al. 2013, El-Sayed et al. 2015). Esto es particularmente impotante porque recientemente este se ha considerado a este ecosistema como posible asiento de un muelle de manejo y transporte de minerales de envergadura continental (Quintero et al. 2006).

\section{MATERIALES Y MÉTODOS}

Las muestras de sedimentos superficiales se recolectaron en doce estaciones (Ests.) (Fig. 1), en abril de 2008, con una draga Diez Laffont de $0.02 \mathrm{~m}^{2}$ de área. Se tomaron siete réplicas de sedimento, en cada punto de muestreo, las cuales fueron homogeneizadas rápidamente. Inmediatamente después de la toma de muestras los sedimentos se mantuvieron en posición vertical, dentro de bolsas de polietileno selladas, cubiertos por bolsas plásticas negras y congelados hasta su análisis (Fuentes et al. 2010).

\section{Concentración total de metales}

Un gramo del sedimento $(<63 \mu \mathrm{m})$ secado al aire y tamizado, se digirió por triplicado en una mezcla de $2 \mathrm{~mL}$ de agua ultrapura y $20 \mathrm{~mL}$ de $\mathrm{HNO}_{3}$ concentrado, durante $12 \mathrm{~h}$, a temperatura ambiente. Luego se

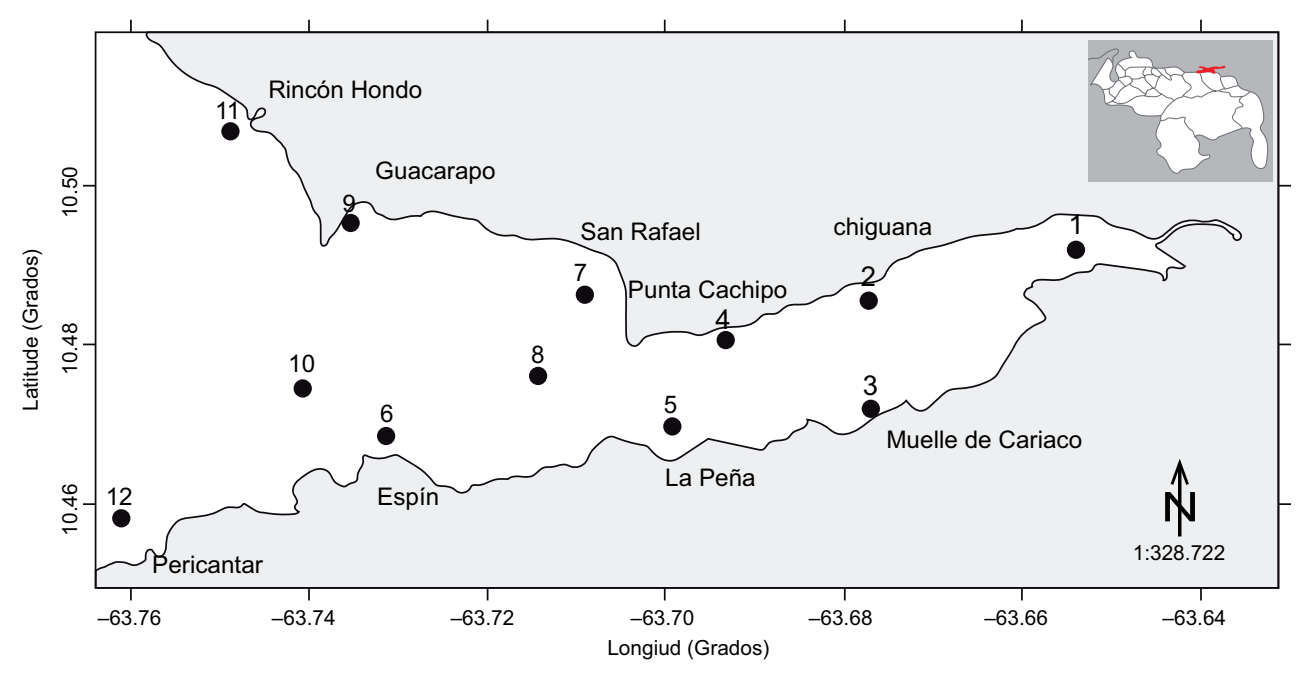

Fig. 1. Ubicación geográfica del saco del golfo de Cariaco y estaciones de muestreo 
calentó a $80^{\circ} \mathrm{C}$, durante $2 \mathrm{~h}$ más. Progresivamente se aumentó la temperatura hasta $120^{\circ} \mathrm{C}$ y se mantuvo en esas condiciones por $2 \mathrm{~h}$. Una vez fría, la muestra se filtró y diluyó hasta $25 \mathrm{~mL}$ (Rojas et al. 2002). La cuantificación de las concentraciones totales de metales se ejecutó con un espectrómetro de emisión óptica con plasma acoplado inductivamente (ICPOES, Perkin Elmer, modelo 5300 DV), el cual se calibró con las siguientes condiciones: flujo de argón al plasma, $15 \mathrm{~L} / \mathrm{min}$; flujo auxiliar de argón, $0.2 \mathrm{~L} /$ min; flujo del nebulizador, $0.8 \mathrm{~L} / \mathrm{min}$; generación de frecuencia, $1300 \mathrm{~W}$; caudal del flujo a la bomba peristáltica, $1.5 \mathrm{~mL} / \mathrm{min}$, y temperatura, $30{ }^{\circ} \mathrm{C}$. Para minimizar el efecto matriz, las soluciones estándares se prepararon con agua de mar artificial filtrada $(0.45$ $\mu \mathrm{m})$. Por separado se preparó una curva de adición de analito para verificar el efecto matriz, el cual fue despreciable. Todos los reactivos y patrones externos de calibración fueron de grado analítico, y los análisis se realizaron por triplicado para verificar la precisión del método de digestión y la recuperación de los metales.

La confiabilidad de la extracción de los metales totales se evaluó con un material de referencia certificado de sedimentos marino (HISS-1). La exactitud del método fue menor a $9 \%$ y la precisión menor a $10 \%$. Los blancos de cada conjunto de muestras estuvieron por debajo del $1 \%$ de los valores de las muestras y la precisión de las muestras menor que $3 \%$; los límites de detección (LD) obtenidos de la curva de calibración (Meier y Zünd 1993) fueron los siguientes: $\mathrm{Fe}, 0.08 \mu \mathrm{g} / \mathrm{g}$; Mn, $0.02 \mu \mathrm{g} / \mathrm{g} ; \mathrm{Cr}, 0.05 \mu \mathrm{g} / \mathrm{g}$; $\mathrm{Zn}, 0.10 \mu \mathrm{g} / \mathrm{g} ; \mathrm{Ni}, 0.03 \mu \mathrm{g} / \mathrm{g} ; \mathrm{Cu}, 0.05 \mu \mathrm{g} / \mathrm{g} ; \mathrm{Cd}$, $0.02 \mu \mathrm{g} / \mathrm{g}, \mathrm{y} \mathrm{Pb}, 0.01 \mu \mathrm{g} / \mathrm{g}$.

\section{Concentración de metales intercambiables y en- lazados a carbonatos}

Las concentraciones de los metales Fe, Mn, Zn, $\mathrm{Cd}, \mathrm{Cr}, \mathrm{Cu}, \mathrm{Ni}$ y $\mathrm{Pb}$ presentes en forma iónica y enlazados a carbonatos (F1) se obtuvieron con el método propuesto por Campanella et al. (1995). A $2.5 \mathrm{~g}$ de sedimento seco homogeneizado $(<63 \mu \mathrm{m})$ se agregaron $45 \mathrm{~mL}$ de acetato de amonio $1 \mathrm{~mol} / \mathrm{L}$, ajustado a pH 5 con ácido acético. Esta mezcla fue agitada durante $24 \mathrm{~h}$ a temperatura ambiente, en un agitador mecánico a $130 \mathrm{rpm}$. Después de la extracción, la mezcla se centrifugó a $3000 \mathrm{rpm}$ durante 20 min. El sobrenadante se removió y colocó en un matraz aforado de $50 \mathrm{~mL}$, donde se enrasó. Los metales se analizaron en dicha solución en el ICP-OES, en las mismas condiciones indicadas anteriormente. Todas las extracciones se ejecutaron por triplicado y la precisión de las réplica fue menor a $3 \%$.

\section{Factor de enriquecimiento}

Para diferenciar la procedencia natural de las contribuciones antrópicas de metales y evaluar su impacto se calculó el factor de enriquecimiento (FE) respecto a la abundancia natural de los metales en la corteza terrestre (Turekian y Wedepohl 1961). Dicho factor reduce la variabilidad de los constituyentes asociada con las diversas relaciones granulométricas; es una herramienta conveniente para graficar tendencias geoquímicas a lo largo de áreas geográficas, e informa sobre la dinámica de un contaminante inorgánico o elemento químico de la corteza terrestre que puede ser acarreado por lluvia, viento o bien tener su origen en fuentes antrópicas (Kamau 2002, del Águila et al. 2005, Bitan et al. 2013). Para normalizar los datos se escogió Fe como elemento conservativo, ya que usualmente presenta altas concentraciones naturales, por lo que su distribución no está relacionada con otros metales pesados y difícilmente es enriquecido por fuentes antrópicas (Deely y Fergusson 1994). Su comportamiento geoquímico es similar a otros metales, tanto en ambientes óxicos como anóxicos, es muy abundante en la zona costera del estado Sucre y las fuentes naturales controlan $98 \%$ de su presencia en el ambiente (Fuentes 1999).

$$
\mathrm{FE}=\frac{\left(\frac{\mathrm{Metal}}{\mathrm{Fe}}\right) \text { Sedimento }}{\left(\frac{\text { Metal }}{\mathrm{Fe}}\right) \text { Corteza terrestre }}
$$

Un valor de FE entre 0.5 y 1.5 sugiere que los metales pueden provenir totalmente de la corteza terrestre o de procesos de meteorización naturales, en tanto que un FE superior a 1.5 indica que una porción de material es aportado por procesos de meteorización no naturales, fuentes distintas a la corteza terrestre o fuentes antrópicas (Zhang y Lui 2002).

\section{Índice de geoacumulación (Igeo)}

Este índice introducido por Müller (1979) permite evaluar la probable contaminación metálica

Igeo $=\log _{2}\left(\frac{C_{n}}{1.5 B_{n}}\right)$

donde $C_{\mathrm{n}}$ es la concentración del metal $(n), B_{\mathrm{n}}$ la concentración geoquímica basal del metal $(n)$ y 1.5 un factor de corrección de los valores basales debido a efectos litogénicos. Las clases de Igeo son siete: no contaminado (Igeo $\leq 0)$, ligeramente contaminado $(0<$ Igeo $\leq 1)$, moderadamente contaminado $(1<$ Igeo $\leq 2)$, 
de moderado a severamente contaminado $(2<$ Igeo $\leq 3)$, severamente contaminado $(3<$ Igeo $\leq 4)$, de severa a extremadamente contaminado $(4<$ Igeo $\leq 5)$ y extremadamente contaminado (Igeo $\geq 5$ ). Esta última clase es al menos 100 veces mayor que la concentración química basal.

\section{Código de riesgo ambiental (CRA)}

El código de riesgo ambiental describe la movilidad potencial de un metal pesado en el sedimento. Es un índice relativo entre las concentraciones de cada metal en las fracciones químicas más débilmente enlazadas al sedimento $\left(\mathrm{C}_{\mathrm{F} 1}\right)$ y la concentración total $\left(\mathrm{C}_{\text {total }}\right)$ de cada metal en el sedimento (Salbu et al. 1988, Ahdy y Youssef 2011).

$\mathrm{CRA}=\left(\frac{C_{F I}}{C_{\text {total }}}\right) \times 100$

Los valores bajos de CRA indican alta estabilidad del metal en el sedimento y por ende poca accesibilidad biológica. Este índice de movilidad y riesgo biológico comprende cinco categorías: sin riesgo $(<1 \%)$, riesgo bajo (1-10\%), riesgo medio $(11-30 \%)$, riesgo alto $(31-50 \%)$ y riesgo muy alto $(>50 \%)$.

\section{Comparación con lineamientos de calidad de sedimentos}

La evaluación de la potencialidad tóxica o riesgo al ecosistema fue realizada por comparación con directrices de calidad de sedimentos (SQG) establecidas (Long et al. 1995, 2006, MacDonald et al. 1996, 2000). Los tres conjuntos SQG adoptados son los siguientes:

1. Niveles de efecto umbral (TEL) y niveles de efectos probables (PEL). Concentraciones por debajo de TEL no están asociadas con ningún efecto biológico adverso, entre TEL y PEL pueden ocurrir ocasionalmente y por encima de PEL frecuentemente (MacDonald et al. 2000).

2. Intervalo de efectos bajos (ERL) e intervalo de efectos medianos (ERM). Concentraciones por debajo del ERL rara vez pueden provocar efectos biológicos adversos $(<10 \%)$, entre ERL y ERM ocasionalmente y superiores a ERM frecuentemente, lo cual corresponde al $50 \%$ de los estudios realizados (Long et al. 1995).

3. Estándares de calidad de sedimentos (SQS) marinos. Criterio de calidad correspondiente a sedimentos no relacionados con efectos adversos sobre los recursos biológicos, incluyendo agudos y crónicos, ni riesgos significativos a la salud humana (WAC 1995).

\section{Cociente ERM promedio}

El efecto concurrente de contaminantes antrópicos múltiples, cuyas concentraciones superan a SQG, se estima mediante el cálculo del cociente medio ERM (Long et al. 1998).

$\mathrm{m}-\mathrm{ERM}-\mathrm{Q}=\frac{\sum_{\mathrm{i}=1}^{\mathrm{n}}\left(\frac{C_{i}}{\mathrm{ERM}_{i}}\right)}{\mathrm{n}}$

$C_{i}$ es la concentración medida del metal, $\mathrm{ERM}_{\mathrm{i}}$ el valor ERM para el metal $i, \mathrm{y} n$ el número de metales $i$. De acuerdo con los valores del cociente medio ERM se definen varias clases de probabilidad tóxica para la biota: $\mathrm{m}-\mathrm{ERM}-\mathrm{Q}<0.10$ (9\%), 0.11-0.50 (21\%), $0.51-1.50(49 \%),>1.50(76 \%)$.

\section{Supervivencia de Artemia}

Para detectar la toxicidad de los posibles contaminantes adheridos a la fase sólida del sedimento se utilizó una modificación de la norma mexicana NMXAA-110-1995-SCFI para la evaluación de la toxicidad aguda con Artemia franciscana (SCFI 1995). A alícuotas de $5 \mathrm{~g}$, pesadas exactamente, se añadieron $15 \mathrm{~mL}$ de diclorometano. La combinación solvente-sedimento se colocó en un baño de ultrasonido (Ultrasonics Transsonic 690 , modelo 9308$)$ a temperatura ambiente $\left(28^{\circ} \mathrm{C}\right)$, durante $15 \mathrm{~min}$. Una vez transcurrido este periodo, la solución fue decantada o retirada con una pipeta. El proceso se repitió en el sedimento remanente y los extractos obtenidos se mezclaron. Posteriormente, los extractos se filtraron y redujeron hasta la sequedad. Luego se añadieron $5 \mathrm{~mL}$ de dimetilsulfóxido al $5 \%$ $\mathrm{v} / \mathrm{v}$ en agua de mar artificial.

Con cada uno de los extractos se preparó una serie de diluciones: 1000,500 y $250 \mu \mathrm{L}$ del extracto completados hasta $2 \mathrm{~mL}$ con agua de mar artificial filtrada, la cual fue usada como control negativo. Se incluyeron también soluciones al $5 \% \mathrm{v} / \mathrm{v}$ de dimetil sulfóxido (DMSO) y diluciones con $15,25,35,45$,

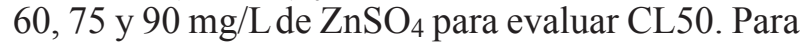
todos los tratamientos, se prepararon cinco réplicas.

En cada dilución y controles fueron colocados 10 nauplios de Artemia salina durante $24 \mathrm{~h}$ con iluminación. Transcurrido este periodo se contaron organismos vivos y muertos.

\section{RESULTADOS}

\section{Concentración total de metales}

Las concentraciones totales de metales estuvieron distribuidas muy uniformemente (Cuadro I). Sus 
CUADRO I. CONCENTRACIÓN TOTAL DE METALES PESADOS ( $\mu \mathrm{g} / \mathrm{g})$ EN LOS SEDIMENTOS SUPERFICIALES DEL SACO DEL GOLFO DE CARIACO

\begin{tabular}{ccccccccc}
\hline Estación & $\mathrm{Fe}\left(10^{3}\right)$ & $\mathrm{Mn}$ & $\mathrm{Zn}$ & $\mathrm{Cd}$ & $\mathrm{Cr}$ & $\mathrm{Cu}$ & $\mathrm{Ni}$ & $\mathrm{Pb}$ \\
\hline 1 & 17 & 47 & 87 & 3.8 & 23 & 90 & 24 & 8 \\
2 & 16 & 44 & 86 & 4.5 & 34 & 72 & 24 & 5 \\
3 & 18 & 62 & 87 & 3.4 & 24 & 60 & 19 & 7 \\
4 & 18 & 56 & 104 & 4.8 & 35 & 91 & 23 & 9 \\
5 & 17 & 54 & 76 & 3.7 & 25 & 57 & 18 & 4 \\
6 & 12 & 32 & 42 & 2.8 & 13 & 33 & 12 & 4 \\
7 & 21 & 49 & 113 & 4.9 & 29 & 47 & 20 & 9 \\
8 & 19 & 63 & 104 & 4.4 & 36 & 84 & 33 & 9 \\
9 & 10 & 19 & 59 & 3.3 & 10 & 35 & 18 & 4 \\
10 & 12 & 28 & 50 & 3.2 & 10 & 62 & 14 & 4 \\
11 & 6 & 12 & 32 & 3.1 & 13 & 33 & 10 & 3 \\
12 & 12 & 19 & 54 & 1.9 & 4 & 29 & 34 & 4 \\
Promedio & 15 & 40 & 75 & 3.7 & 21 & 58 & 21 & 6 \\
Desvest. & 4 & 18 & 27 & 0.9 & 11 & 23 & 7 & 2 \\
LD & 0.08 & 0.02 & 0.1 & 0.04 & 0.05 & 0.05 & 0.03 & 0.01 \\
\hline LD: Límite de detección & & & & & & &
\end{tabular}

valores máximos se presentaron en un paralelogramo regional, cuyos vértices son: centro (Est. 8), norte (Est. 7, San Rafael), oriente (Est. 1) y sur (Est. 3, muelle de Cariaco) e incluye estaciones de la costa norte (Ests. 1, 2, 4, 7). Fuera del perímetro, las concentraciones fueron menores, en particular hacia el área de la Peña (Est. 5), Espín (Est. 6) y Pericantar (Est. 12), ubicadas en la costa sur.

Las concentraciones de metales fueron menores que las referencias geoquímicas de la fracción limo y arcilla (Krupadam et al. 2006), similares al límite inferior de los intervalos de concentraciones informadas por Martínez (2002) y Velásquez (2005) en la misma zona, excepto las concentraciones de $\mathrm{Pb}$, que fueron cuatro veces menores en este estudio. En la costa norte, las concentraciones resultaron más bajas que los contenidos informados por Márquez et al. (2005) y no mostraron diferencias significativas con los valores obtenidos por Fuentes et al. (2010). Además, las correlaciones ( $\mathrm{r}$ ) de Pearson mostraron las siguientes afinidades: Fe- $\mathrm{Zn}=0.95, \mathrm{Fe}-\mathrm{Mn}=$ $0.93, \mathrm{Zn}-\mathrm{Mn}=0.86$, Fe-metal $>0.65, \mathrm{Zn}$-metal $\geq$ 0.83 , Mn-metal $>0.73$ (Cuadro II).

\section{Concentración de metales intercambiables y aso- ciados con carbonatos (F1)}

En esta forma química $\mathrm{Pb}$ estuvo por debajo del límite detección ( $<\mathrm{LD})$ y Cd no fue detectado (ND). En la figura 2 se muestra la distribución de sus concentraciones, las cuales variaron de la siguiente manera: Fe: 50-350 $\mu \mathrm{g} / \mathrm{g}, 100 \pm 82 \mu \mathrm{g} / \mathrm{g}$; Cr: < LD$0.60 \mu \mathrm{g} / \mathrm{g}, 0.36 \pm 0.20 \mu \mathrm{g} / \mathrm{g}$; Ni: ND-1.23 $\mu \mathrm{g} / \mathrm{g}, 0.66$
CUADRO II. COEFICIENTE DE CORRELACIÓN DE PEARSON

\begin{tabular}{lcccccccc}
\hline & $\mathrm{Fe}$ & $\mathrm{Mn}$ & $\mathrm{Zn}$ & $\mathrm{Cd}$ & $\mathrm{Cr}$ & $\mathrm{Cu}$ & $\mathrm{Ni}$ & $\mathrm{Pb}$ \\
\hline $\mathrm{Fe}$ & 1.00 & 0.93 & 0.94 & 0.70 & 0.78 & 0.64 & 0.45 & 0.84 \\
$\mathrm{Mn}$ & & 1.00 & 0.85 & 0.68 & 0.85 & 0.73 & 0.34 & 0.77 \\
$\mathrm{Zn}$ & & & 1.00 & 0.83 & 0.86 & 0.69 & 0.52 & 0.91 \\
$\mathrm{Cd}$ & & & & 1.00 & 0.91 & 0.67 & 0.11 & 0.73 \\
$\mathrm{Cr}$ & & & & & 1.00 & 0.76 & 0.31 & 0.75 \\
$\mathrm{Cu}$ & & & & & & 1.00 & 0.34 & 0.70 \\
$\mathrm{Ni}$ & & & & & & & 1.00 & 0.47 \\
$\mathrm{~Pb}$ & & & & & & & & 1.00 \\
\hline
\end{tabular}

$\pm 0.35 \mu \mathrm{g} / \mathrm{g} ; \mathrm{Cu}:<\mathrm{LD}-8 \mu \mathrm{g} / \mathrm{g}, 3 \pm 3 \mu \mathrm{g} / \mathrm{g} ; \mathrm{Zn}: 4-27$ $\mu \mathrm{g} / \mathrm{g}, 13 \pm 8 \mu \mathrm{g} / \mathrm{g} ; \mathrm{Mn} ;<\mathrm{LD}-16 \mu \mathrm{g} / \mathrm{g}, 7 \pm 3 \mu \mathrm{g} / \mathrm{g}$. En el ecosistema, Cr fue medible en $42 \%$ (Ests. 1, $3,5,7,9$ ), mientras que $\mathrm{Ni}$ en $50 \%$ (Ests. 1, 3, 5, 7, 9,12 ) y $\mathrm{Cu}$ en $75 \%$ (Ests. 1-5, 9-12).

\section{Factor de enriquecimiento}

La figura 3 muestra que el factor de enriquecimiento (FE) de Mn estuvo entre 0.14 y 0.28 (0.24 $\pm 0.05)$, Cr entre 0.17 y $1.13(0.74 \pm 0.29), \mathrm{Pb}$ entre 0.55 y $1.17(0.92 \pm 0.36)$, Ni entre 0.73 y $1.96(1.00$ $\pm 0.30), \mathrm{Zn}$ entre 1.73 y $2.92(2.47 \pm 0.35), \mathrm{Cu}$ entre 2.34 y $5.75(4.14 \pm 1.22)$ y Cd entre 24 y $82(41 \pm$ 15). La distribución regional del $\mathrm{FE}$ fue uniforme para todos los metales, excepto Cd que mostró un enriquecimiento mayor en Guacarapo (Est. 9), Rincón Hondo (Est. 10) y localidades vecinas, y Cu, con máximos en la costa norte. De acuerdo con el valor 


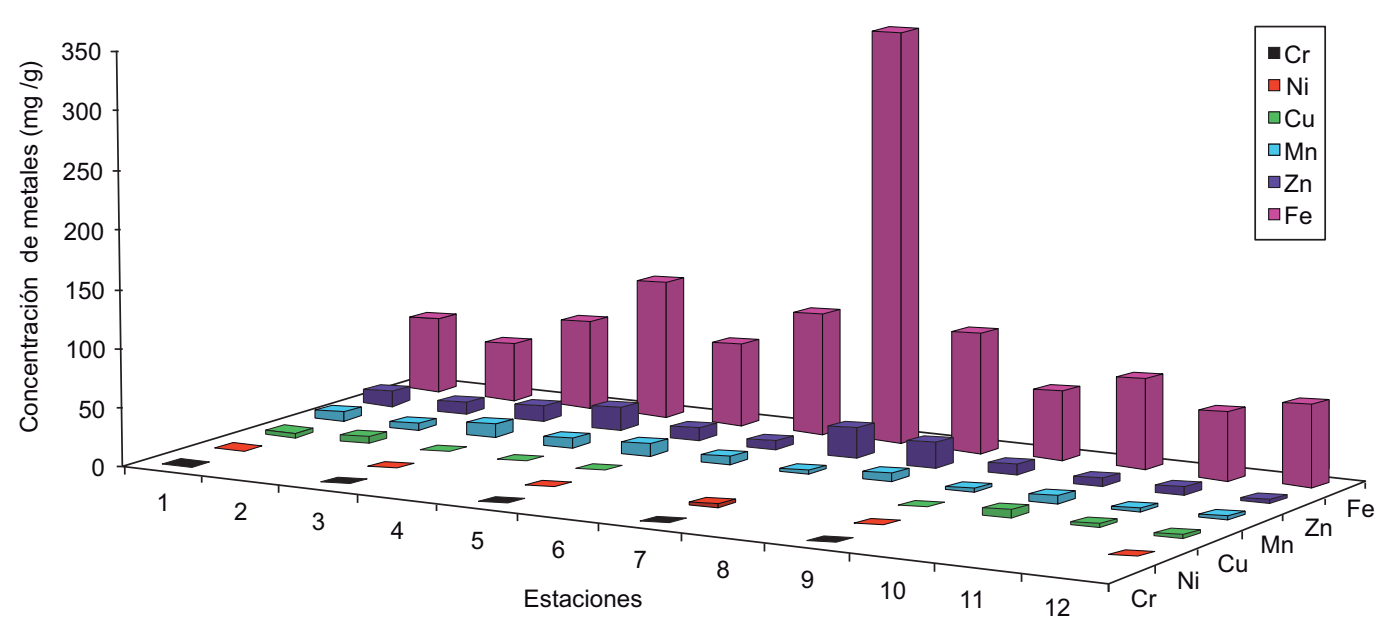

Fig. 2. Concentración de metales intercambiables y asociados con carbonatos en los sedimentos superficiales del saco del golfo de Cariaco (Cd no detectado, $\mathrm{Pb}$ por debajo del límite de detección)

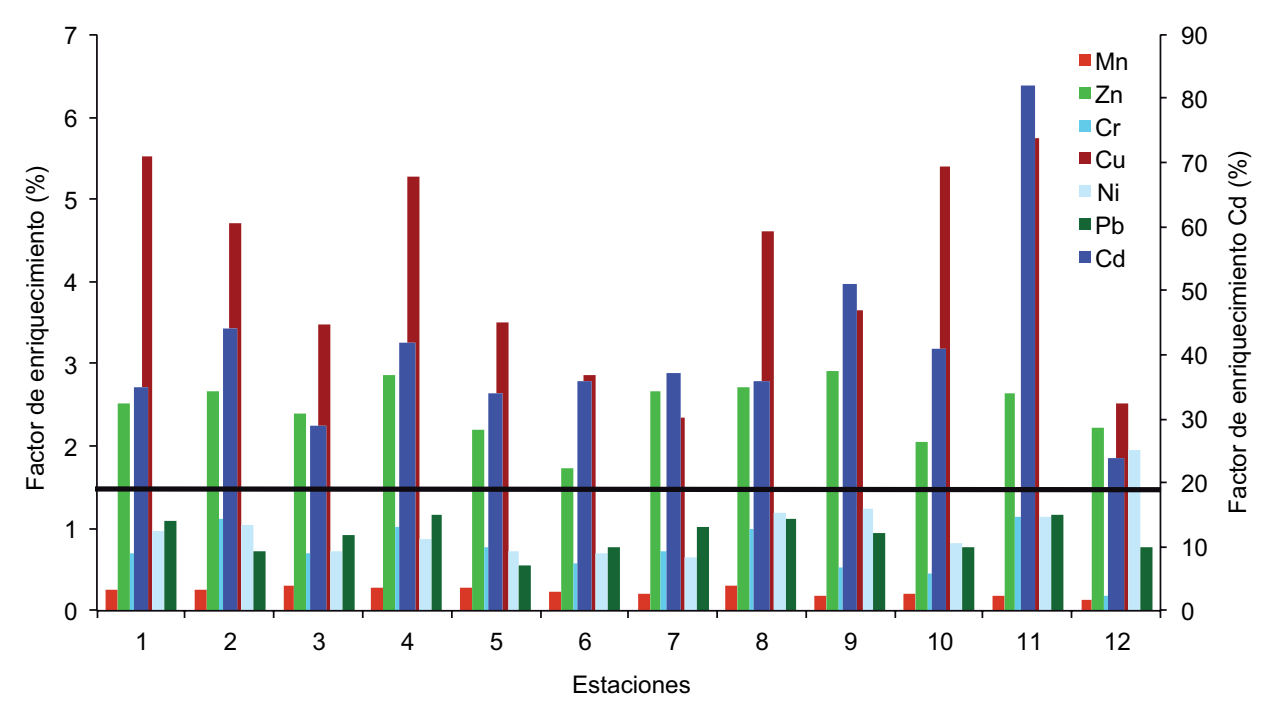

Fig. 3. Factor de enriquecimiento en los sedimentos superficiales del saco del golfo de Cariaco

calculado, los factores de enriquecimiento resultaron menores que 1.5 para $\mathrm{Mn}, \mathrm{Cr}, \mathrm{Pb}, \mathrm{Ni}$; sin embargo, para $\mathrm{Zn}, \mathrm{Cd}, \mathrm{Cu}$ superaron esa cifra.

\section{Índice de geoacumulación}

El Igeo varió de la siguiente manera: $\mathrm{Cr}(-5.08$ a $-1.91,-2.90 \pm 0.97), \mathrm{Pb}(-3.32 \mathrm{a} 1.74,-2.52 \pm 0.58)$, $\mathrm{Ni}(-3.35 \mathrm{a}-1.58,-2.38 \pm 0.53), \mathrm{Zn}(-1.40 \mathrm{a}-0.33$, $-1.03 \pm 0.58), \mathrm{Cu}(-1.22$ a $0.43,-0.34 \pm 0.60), \mathrm{Cd}$ (2.06 a 3.46, $2.98 \pm 0.39)$. Estos resultados muestran tendencias regionales muy similares (Fig. 4) pero incrementos distintos. Nótese que $\mathrm{Cd}$, es el único metal con Igeo superior a cero, en toda el área, y $\mathrm{Cu}$ en la zona más interna (Ests. 1, 2, 4) y centro (Est. 8).

\section{Código de riesgo ambiental}

El porcentaje de metales lábiles (F1) respecto a la concentración total, expresada como CRA (Fig. 5), presentó los siguientes intervalos: Fe (0.30-0.90\%, $0.70 \pm 0.40 \%), \mathrm{Cr}(0-2 \%, 2 \pm 1 \%), \mathrm{Ni}(0-6 \%, 3 \pm$ $2 \%), \mathrm{Cu}(0-13 \%, 6 \pm 5 \%), \mathrm{Zn}(7-24 \%, 16 \pm 5 \%)$, Mn (6-26\%, $18 \pm 6 \%)$. Estos resultados distinguen tres grupos con movilidades potenciales distintas. El primer grupo incluye $\mathrm{Fe}, \mathrm{Cd}, \mathrm{Pb}, \mathrm{Cr}$, $\mathrm{Ni}$ (Ests. 2, 4, 6, 


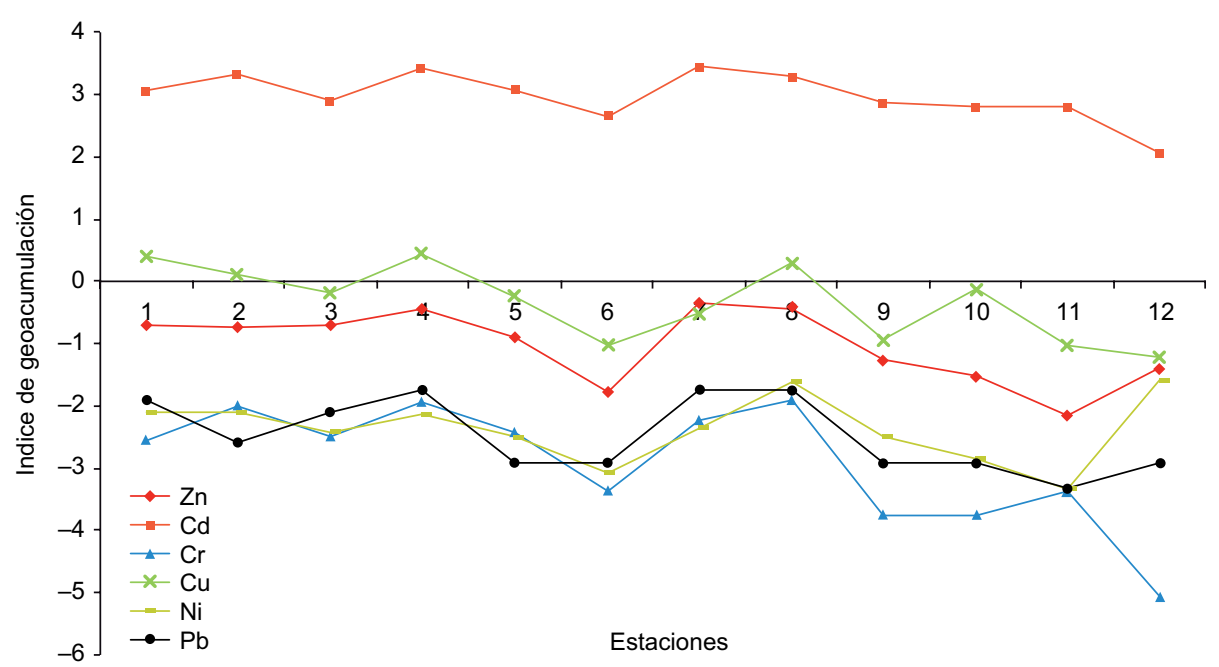

Fig. 4. Índice de geoacumulación en los sedimentos superficiales del saco del golfo de Cariaco

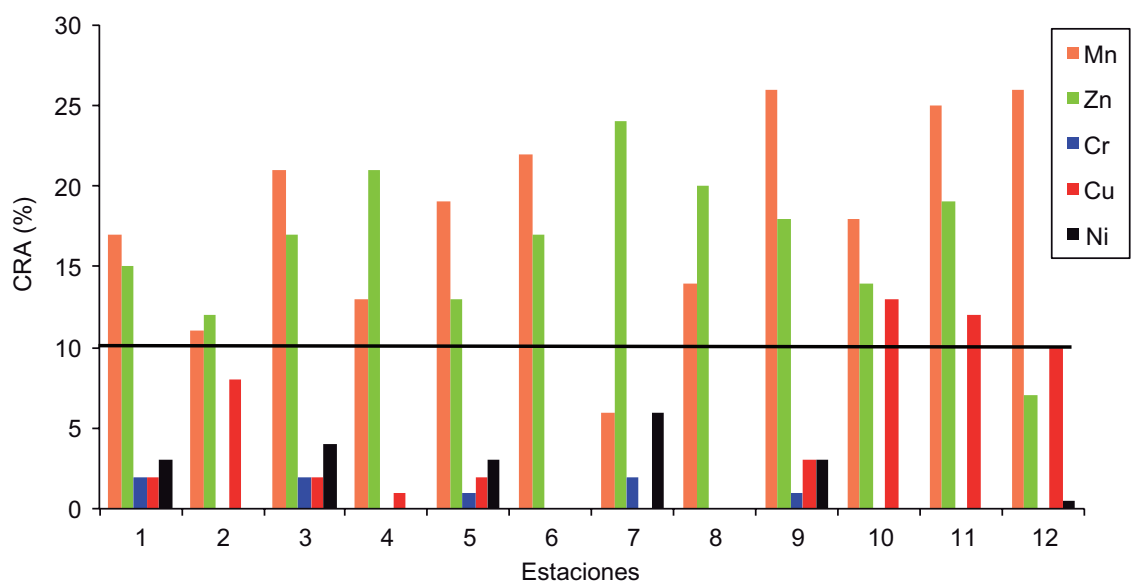

Fig. 5. Código de riesgo ambiental (CRA) en los sedimentos superficiales del saco del golfo de Cariaco

$8,10-12$ ) y $\mathrm{Cu}$ (Ests. 6-8) con CRA menor que $1 \%$; el segundo grupo contiene Mn (Est. 7), Zn (Est. 12), $\mathrm{Cr}$ (Ests. 1, 3, 5, 7, 9), Cu (Ests. 1-5, 9, 12) y Ni (Ests. $1,3,5,7,9)$ con CRA entre 1 y $10 \%$, y el tercer grupo comprende Mn (Ests. 1-6, 8-12), Zn (Ests. 1-11) y Cu (Ests. 10, 11) con CRA entre 11 y $30 \%$.

\section{Comparación con los lineamientos de calidad de sedimento}

Los resultados del contraste de las tres directrices de calidad de sedimento con las concentraciones de los metales se muestran en el cuadro III. Todas las muestras presentaron concentraciones de $\mathrm{Cr}, \mathrm{Pb}$ y $\mathrm{Zn}$ menores que TEL y ERL, mientras que $25 \%$ de las muestras registraron concentraciones de Ni menores que sus correspondientes TEL (Ests. 6, 10, 11) y $58 \%$ de ellas inferiores a su ERL (Ests. 3, 5-7, 9-11).
$\mathrm{Cu}$ fue menor que ERL en $17 \%$ (Ests. 6, 11) de las muestras estudiadas. Respecto del segundo nivel establecido por los criterios de calidad (TEL-PEL, ERL-ERM), $\mathrm{Cd}$, $\mathrm{Ni}$ y $\mathrm{Cu}$ fueron menores que PEL (ERM) en $67 \%$ (100\%), $75 \%$ (42\%) y $100 \%$ (83\%) del ecosistema. Y solamente Cd superó PEL en $33 \%$ de las muestras (Ests. 2, 4, 7, 8). Finalmente, ninguna muestra superó los SQS establecidos por el estado de Washington (WAC 1995).

\section{Cociente promedio ERM}

Al calcular la distribución espacial del riesgo potencial multimetálico en el saco del golfo de Cariaco se encontraron valores entre 0.13 (Est. 6) y 0.30 (Est. 8), con máximos (0.25-0.30) en la costa norte (Ests. 2, 4, 7) y centro (Est. 8), mayoritariamente influenciados por las relaciones $C_{\mathrm{i}} / E R M_{\mathrm{i}}$ de $\mathrm{Cd}$, $\mathrm{Ni}$ y $\mathrm{Cu}$ (Cuadro IV). 
CUADRO III. COMPARACIÓN ENTRE LAS CONCENTRACIONES DE METALES PESADOS $(\mu \mathrm{g} / \mathrm{g})$ EN LOS SEDIMENTOS DEL SACO DEL GOLFO DE CARIACO Y LAS DIRECTRICES PARA LACALIDAD DE SEDIMENTOS (SQG) CON EL PORCENTAJE DE MUESTRAS EN CADA DIRECTRIZ

\begin{tabular}{|c|c|c|c|c|c|c|}
\hline \multirow{2}{*}{$\begin{array}{l}\text { Directrices de calidad } \\
\text { para los sedimentos }\end{array}$} & \multicolumn{6}{|c|}{ Concentración $(\mu \mathrm{g} / \mathrm{g})$} \\
\hline & $\mathrm{Zn}$ & $\mathrm{Cd}$ & $\mathrm{Cr}$ & $\mathrm{Cu}$ & $\mathrm{Ni}$ & $\mathrm{Pb}$ \\
\hline TEL & 124 & 0.6 & 52.3 & 18.7 & 15.9 & 30.2 \\
\hline PEL & 271 & 4.2 & 160.4 & 108.2 & 42.8 & 112.2 \\
\hline ERL & 150 & 1.2 & 81 & 34 & 20.9 & 46.7 \\
\hline ERM & 410 & 9.6 & 370 & 270 & 51.6 & 218 \\
\hline SQS & 410 & 5.1 & 260 & 390 & & 450 \\
\hline \multicolumn{7}{|c|}{ Comparada con TEL y PEL } \\
\hline$<\mathrm{TEL}$ & $100 \%$ & $0 \%$ & $100 \%$ & $0 \%$ & $25 \%$ & $100 \%$ \\
\hline $\mathrm{TEL} \leq C_{\mathrm{i}}<\mathrm{PEL}$ & $0 \%$ & $67 \%$ & $0 \%$ & $100 \%$ & $75 \%$ & $0 \%$ \\
\hline$\geq$ PEL & $0 \%$ & $33 \%$ & $0 \%$ & $0 \%$ & $0 \%$ & $0 \%$ \\
\hline \multicolumn{7}{|c|}{ Comparada con ERL Y ERM } \\
\hline$<\mathrm{ERL}$ & $100 \%$ & $0 \%$ & $100 \%$ & $17 \%$ & $58 \%$ & $100 \%$ \\
\hline $\mathrm{ERL} \leq C_{\mathrm{i}}<\mathrm{ERM}$ & $0 \%$ & $100 \%$ & $0 \%$ & $83 \%$ & $42 \%$ & $0 \%$ \\
\hline$\geq \mathrm{ERM}$ & $0 \%$ & $0 \%$ & $0 \%$ & $0 \%$ & $0 \%$ & $0 \%$ \\
\hline \multicolumn{7}{|l|}{ Comparada con SQS } \\
\hline$<\mathrm{SQS}$ & $100 \%$ & $100 \%$ & $100 \%$ & $100 \%$ & & $100 \%$ \\
\hline
\end{tabular}

TEL: niveles de efecto umbral, PEL: niveles de efectos probables, ERL: intervalo de efectos bajo, ERM: intervalo de efectos mediano; SQS: calidad estándar

\section{Supervivencia de Artemia}

Ninguna larva de Artemia salina murió después ser expuestas a diluciones del extracto del sedimento, preparadas de manera tal que estuvieran en contacto con la cantidad de sustancias presentes en $0.25,0.5 \mathrm{y}$ $1 \mathrm{~g}$ de sedimento, en el tiempo de desarrollo larvario correspondiente a $24 \mathrm{~h}$.

\section{DISCUSIÓN}

Las concentraciones totales de los metales sugieren que los sedimentos son, principalmente, detritos de suelos y rocas meteorizadas, los cuales han sufrido cambios diagenéticos y contienen una pequeña cantidad de metales antrópicos (Krupa-

CUADRO IV. COCIENTE PROMEDIO DE LOS VALORES DEL INTERVALO DE EFECTOS MEDIANOS (m-ERM-Q) DE LOS SEDIMENTOS SUPERFICIALES DEL SACO DEL GOLFO DE CARIACO

\begin{tabular}{lccccccc}
\hline \multirow{2}{*}{ Estaciones } & \multicolumn{7}{c}{ Ci/ERMi } \\
\cline { 2 - 8 } & $\mathrm{Zn}$ & $\mathrm{Cd}$ & $\mathrm{Cr}$ & $\mathrm{Cu}$ & $\mathrm{Ni}$ & $\mathrm{Pb}$ & m-ERM-Q \\
\hline 1 & 0.21 & 0.40 & 0.06 & 0.33 & 0.47 & 0.04 & 0.25 \\
2 & 0.21 & 0.47 & 0.09 & 0.27 & 0.47 & 0.02 & 0.25 \\
3 & 0.21 & 0.35 & 0.06 & 0.22 & 0.37 & 0.03 & 0.21 \\
4 & 0.25 & 0.50 & 0.09 & 0.34 & 0.45 & 0.04 & 0.28 \\
5 & 0.19 & 0.39 & 0.07 & 0.21 & 0.35 & 0.02 & 0.20 \\
6 & 0.10 & 0.29 & 0.04 & 0.12 & 0.23 & 0.02 & 0.13 \\
7 & 0.28 & 0.51 & 0.08 & 0.17 & 0.39 & 0.04 & 0.25 \\
8 & 0.25 & 0.46 & 0.10 & 0.31 & 0.64 & 0.04 & 0.30 \\
9 & 0.14 & 0.34 & 0.03 & 0.13 & 0.35 & 0.02 & 0.17 \\
10 & 0.12 & 0.33 & 0.03 & 0.23 & 0.27 & 0.02 & 0.17 \\
11 & 0.08 & 0.33 & 0.04 & 0.12 & 0.19 & 0.01 & 0.13 \\
12 & 0.13 & 0.20 & 0.01 & 0.11 & 0.66 & 0.02 & 0.19 \\
\hline
\end{tabular}


dam et al. 2006). Este planteamiento es soportado por las fuertes correlaciones exhibidas entre tres metales naturales de la litósfera (Fe, $\mathrm{Mn}, \mathrm{Zn}$ ) y los restantes metales (García-Rico et al. 2004). Por otro lado, Rubio et al. (2000) señalan que una gran cantidad de $\mathrm{Fe}, \mathrm{Mn}$ y $\mathrm{Zn}$ en la zona costera son, a priori, de origen litogénico, e indican que su importancia radica en su interacción con los demás metales pesados, más que por su carácter tóxico. Además, MacDonald et al. (1991) resaltan que las correlaciones poco significativas entre $\mathrm{Fe}, \mathrm{Mn}$ y $\mathrm{Zn}$ con otros metales indican la existencia de residuos generados por el hombre. De hecho, Quintero et al. (2005) han informado sobre la meteorización física y química de los suelos de la península de Araya, que junto con los suelos húmicos, arrastrados por el río Carinicuao, constituyen los sedimentos del saco. Aparentemente, la mitad más oriental del saco acumula la mayor cantidad de metales, debido a que en esa porción geográfica los aportes del Carinicuao son directos y arrastran materia orgánica, la cual es un sitio de enlace muy favorable para contaminantes metálicos (Simpson et al. 2005).

Respecto a las referencias regionales, la concentración de metales ha sido constante en la última década. Un aspecto relevante es la disminución del $\mathrm{Pb}$ por la sustitución de la gasolina plomada. De esta manera se redujeron las emanaciones atmosféricas, producto del tráfico automotor. Hung y Hsu (2004) señalan que en los ecosistemas costeros, la fuente de $\mathrm{Pb}$ más importante son los residuos de combustibles liberados hacia la atmósfera por el tráfico flotante y rodante, así como las aguas de desecho liberadas al ecosistema.

El hecho de que porcentajes muy bajos de estas concentraciones totales correspondan a metales en forma de iones intercambiables y precipitados como carbonatos o adsorbidos sobre ellos puede interpretarse como que hay muy pocos metales potencialmente biodisponibles y las actividades antrópicas desarrolladas en el área son de bajo impacto. Rubio et al. (1995) sostienen que en estas formas químicas, los metales son más biodisponibles y generalmente corresponden a aportes antrópicos. Estas especies químicas libres penetran fácilmente las membranas de vegetales y animales y provocan un amplio espectro de efectos tóxicos, especialmente en la etapa larvaria de especies pequeñas, y sólo pueden ser liberados del sedimento por procesos de intercambio debidos a pequeños cambios ambientales, o al descender el $\mathrm{pH}$ del sedimento.

El factor de enriquecimiento permite diferenciar la procedencia antrópica y/o natural de los metales y evaluar el impacto. Un valor de FE entre 0.5 y 1.5 sugiere que los metales pueden provenir totalmente de la corteza terrestre o de procesos de meteorización naturales, mientras que un FE superior a 1.5 indica que una porción de material es aportado por una fuente adicional a la corteza terrestre o por fuentes antrópicas (Zhang y Lui 2002). En consecuencia, las concentraciones de $\mathrm{Mn}, \mathrm{Pb}, \mathrm{Cr}$ y Ni sugieren que estos metales provienen de las rocas sedimentarias de las serranías de la costa sur y el conjunto de rocas sedimentarias y metamórficas de la península de Araya (Caraballo 1982); sin embargo, existen fuentes adicionales de $\mathrm{Zn}, \mathrm{Cu}$ y $\mathrm{Cd}$ que provocan un enriquecimiento menor de $\mathrm{Zn}$, moderado de $\mathrm{Cu}$ y extremadamente severo de $\mathrm{Cd}$ (Sutherland 2000), los cuales pudieron ser arrastrados por vientos, lluvias, el río Carinicuao y/o quebradas y cuya deposición es favorecida por la presencia de corrientes débiles y suave oleaje. Este comportamiento es similar al observado por Martínez (2016), quien señaló que en el saco del golfo de Cariaco es evidente un enriquecimiento menor para la mayoría de los metales, con la excepción de $\mathrm{Cd}$, que presenta un enriquecimiento moderadamente severo. Por ello, esta región del golfo está menos impactada por actividades antrópicas.

Fuentes adicionales de $\mathrm{Cd}, \mathrm{Cu}$ y Ni pueden ser el lavado de suelos agrícolas y las descargas urbanas. Al respecto, Martínez $(2002,2016)$ informó sobre el uso de pesticidas, herbicidas y abonos para las actividades agrícolas desarrolladas en la región de Cariaco, cuyos residuos pueden ser arrastrados al área por el río Carinicuao. Por otro lado, algunos investigadores como Páez-Osuna y Osuna-López (1992) y González-Lozano et al.(2006) han informado que las concentraciones de $\mathrm{Cd}$ pueden estar ligadas a un proceso metabólico del fitoplancton, asociado con altas concentraciones de nutrientes en aguas frías propias de zonas de surgencia; y añaden que el aporte de los metales $\mathrm{Zn}, \mathrm{Cu}$ y $\mathrm{Pb}$ puede ocurrir durante la quema de madera e incineración de basura, actividades frecuentemente practicadas en la zona. Según García et al. (2004), el Cd es fijado por el fitoplancton en la columna de agua y depositado en el fondo marino, donde posteriormente ocurre la descomposición del detritus orgánico y la liberación del metal. El Igeo indica que los sedimentos no están contaminados por $\mathrm{Cr}, \mathrm{Ni}, \mathrm{Pb}$ y Zn, excepto muy moderadamente por $\mathrm{Cu}$ en la parte más interna (Ests. 1, 2, 4) y centro del ecosistema (Est. 8 ), y entre moderada y severamente por Cd en toda la región. Es decir, ambos índices evaluadores relacionan al Cd con un alto grado de contaminación. 
Ling y Yang (2008) indican que el Igeo relaciona, teóricamente, toda la contribución contaminante al valor basal de la corteza, sin diferenciar el efecto litogénico natural de la contribución antrópica. En zonas con meteorización, la roca puede liberar contenidos adicionales de metales y sobrestimar el efecto contaminante.

En los sedimentos, los metales presentes como iones intercambiables y precipitados como carbonatos o adsorbidos en ellos son liberados fácilmente como iones libres durante el proceso de intercambio iónico, debido a pequeños cambios ambientales (potencial redox, salinidad, removilización por corrientes, remoción de los sedimentos por organismos), o al descender el $\mathrm{pH}$ de los sedimentos; en consecuencia, son los más biodisponibles porque pueden introducirse fácilmente en las membranas de los organismos y se relacionan con contaminación antrópica, por ello se consideran en el CRA (Ahdy y Youssef 2011, Hu et al. 2013, Yang et al. 2014). De acuerdo con este criterio, el saco del golfo de Cariaco no presenta riesgo ambiental referente a Fe, $\mathrm{Cd}, \mathrm{y} \mathrm{Pb}$. Cr mostró un riesgo ambiental entre nulo y bajo, $\mathrm{Cu}$ entre nulo y medio, mientras que $\mathrm{Mn}$ y $\mathrm{Zn}$ representan un riesgo potencial medio. Estos resultados no representan un problema grave para el ecosistema porque dichos elementos se encuentran en muy bajas concentraciones. Situación diferente encontró Martínez (2016) en el golfo de Cariaco, quien señala que los metales con mayor CRA son $\mathrm{Pb}, \mathrm{Cd}$ y $\mathrm{Zn}$, debido a residuos agrícolas y al tráfico automotor; sin embargo, los CRA de $\mathrm{Pb}$ y $\mathrm{Cd}$ encontrados son muy parecidos a los registrados para la plataforma norte de la península de Paria, estado Sucre (Venezuela), los cuales no representan un riesgo para el ambiente, pero distintos respecto a $\mathrm{Cu}, \mathrm{Ni}, \mathrm{Cr}$ con riesgos altos. Cabe destacar que la plataforma norte es una región abierta al mar Caribe, y tiene una fuerte hidrodinámica, alta productividad primaria debido a la surgencia y a los aportes sedimentarios de los ríos Orinoco y Amazonas.

El criterio de calidad para sedimentos marinos ERL indica que las concentraciones de $\mathrm{Zn}, \mathrm{Cr}$ y $\mathrm{Pb}$ no tienen la capacidad de provocar daños a los organismos, en tanto que PEL y ERM indican que solamente las concentraciones de $\mathrm{Cd}, \mathrm{Cu}$ y $\mathrm{Ni}$ acumulados, en determinada porción del ecosistema, pudieran provocar daños adversos a los organismos bénticos y epibénticos expuestos directamente, y perjudicar su salud; sin embargo, esta probabilidad de ocurrencia es muy ocasional (MacDonald et al. 2000, Long et al. 1995). Aunque el Cd superó el criterio PEL en $33 \%$ del saco, asociado con una expectativa de ocurrencia de eventos adversos de más de $50 \%$, el ERM no fue superado, sugiriendo que la probabilidad de que ocurran frecuentemente es baja. Y, según al criterio SQS, en toda el área geográfica la probabilidad de consecuencias adversas es nula para los recursos biológicos y humanos. La combinación de los valores SQG específicos de cada metal en la determinación del posible efecto biológico multimetálico, mediante el cociente ERM promedio (m-ERM-Q), establece que un riesgo de toxicidad multimetálico en el saco del golfo de Cariaco superior a 0.10 y menor que 0.30 determinaría una probabilidad potencial tóxica de $21 \%$, con el mayor potencial ecotoxicológico en la zona centro-oriental, donde resultaron muy influyentes $\mathrm{Cd}$ y Ni. Sin embargo, esta probabilidad puede considerarse despreciable, lo cual concuerda con los resultados de la comparación con los criterios de calidad del sedimento y la prueba de supervivencia de Artemia salina.

\section{CONCLUSIONES}

Los metales provienen fundamentalmente de los detritos de suelos y rocas meteorizadas naturalmente y enriquecidos con muy poca contribución antrópica, excepto en el $\mathrm{Cd}$, único metal que mostró enriquecimiento muy severo. Su alto índice de geoacumulación permitió catalogar al ecosistema como un ambiente entre moderado y muy contaminado por $\mathrm{Cd}$, lo cual influye (junto con $\mathrm{Cu}$ y $\mathrm{Ni}$ ) en la probabilidad de ocurrencia de efectos adversos para los recursos biológicos y humanos; sin embargo, la probabilidad tóxica conjunta de los metales es muy baja. La importancia de este riesgo ambiental disminuye debido a la poca cantidad de metales en la fracción lábil, donde el Cd no fue detectado y el $\mathrm{Pb}$ está ausente. El riesgo potencial de toxicidad es insignificante, comprobado por las pruebas toxicológicas que indicaron que no existe una amenaza concreta de daño por metales pesados en los sedimentos del saco del golfo de Cariaco, ni posibles efectos en las condiciones ambientales. Esto es imperativo para asegurar su sostenibilidad futura.

\section{AGRADECIMIENTOS}

Los autores agradecen al Instituto de Investigaciones de Biomedicina y Ciencias Aplicadas (IIBCA), al Instituto Oceanográfico de Venezuela (IOV) y al Departamento de Química de la Universidad de Oriente por el apoyo brindado para la realización de este estudio. 


\section{REFERENCIAS}

Ahdy H. y Youssef D.H. (2011). Fractionation analysis of some metals in sediments of the north-western part of the Red Sea. Egypt. Chem. Ecol. 27 (5), 427-443. DOI: $10.1080 / 02757540.2010 .547488$

Bangqi H., Li J., Zhao J., Yang J., Bai F. y Dou Y. (2013). Heavy metal in a surface sediments of the Liadong Bay Bohai Sea: Distribution, contamination and sources. Environ. Monit. Asses. 185 (6), 5071-5083.

DOI: $10.1007 / \mathrm{s} 10661-012-2926-0$

Bitan A., Kabir S., Selim A., Nazim M. y Ahsan A. (2013). Enrichment factor and geoaccumulation index of trace metals in sediments of the ship breaking area of Sitakund Upazilla (Bhatiary-Kumira), Chittangong, Bangladesh. J. Geochem. Explor. 125, 130-137.

DOI: 10.1016/j.gexplo.2012.12.002

Calderón C. y Valdés J. (2012). Contenido de metales en sedimentos y organismos de la Bahía de San Jorge, Antofagasta, Chile. Rev. Biol. Mar. Oceanogr. 47 (1), 121-133. DOI: $10.4067 / \mathrm{S} 0718-19572012000100011$

Campanella L., D’Orazio D., Petronio B. y Pietrantonio E. (1995). Proposal for a metal speciation study in sediments. Anal. Chim. Acta 309 (1-3), 387-393. DOI: 10.1016/0003-2670(95)00025-U

Caraballo L.F. (1982). Golfo de Cariaco. Parte II: Los sedimentos superficiales y su distribución por el fondo. Fuente de sedimentos. Análisis mineralógico. Bol. Inst. Oceanogr. Venezuela Univ. Oriente 21 (1-2), 37-65.

Deely J.M. y Fergusson J.E. (1994). Heavy metal and organic matter concentration and distributions in dated sediments of a small estuary adjacent to a small urban area. Sci. Total Environ. 153 (1-2), 97-111.

DOI: 10.1016/0048-9697(94)90106-6

Del Águila J., Lugo J. y Vaca R. (2005). Determinación de factor de enriquecimiento y geoacumulación de $\mathrm{Cd}$, $\mathrm{Cr}, \mathrm{Cu} \mathrm{Ni}, \mathrm{Pb}$ y $\mathrm{Zn}$ en suelos de la cuenca alta del río Lerma. CES 12 (2), 155-161 [en línea] https://cienciaergosum.uamex.mx/article/view/7536 06/02/2017

El Nemr A., Khaled A., Moeer A. y El-Skalily A. (2012). Risk probability due to heavy metals en bivalve from eqyptian Mediterreanean. Egypt. J. Aquat. Rev. 38 (2), 67-75. DOI: 10.1016/j.ejar.2012.11.001El-Sayed S.A., Moussa E.M. y El-Saba M.E. (2015). Evaluation of heavy metal content in Qaroun lake, El-Fayoum, Egypt. Part I: Bottom sediments. J. Radiat. Res. Appl. Sci. 8 (3), 276-283. DOI: 10.1016/j.jrras.2015.02.011

Fuentes M.V. (1999). Metales traza en los sedimentos superficiales de la Laguna de Chacopata, Estado Sucre, Venezuela. Scientia 14 (1), 97-116.

Fuentes M.V., Rojas L., Díaz A. y Martínez G. (2010). Distribución de metales pesados en los sedimentos superficiales del Saco del Golfo de Cariaco, Sucre,
Venezuela. Rev. Biol. Trop. 58 (Suppl. 3), 129-140.

García-Rico L., Soto M., Jara M. y Gómez A. (2004). Fracciones geoquímicas de $\mathrm{Cd}, \mathrm{Cu}$ y $\mathrm{Pb}$ en sedimentos costeros de zonas ostrícolas del Estado de Sonora, México. Rev. Intern. Contam. Ambie. 20 (4), 159-167. González-Lozano M., Méndez-Rodríguez D., LópezVeroni D. y Vázquez-Botello A. (2006). Evaluación de la contaminación en sedimentos del área portuaria y zona costera de Salina Cruz, Oaxaca, México. Interciencia 31 (9), 1-23.

Hu B., Liu M., Yang Y., Ou D., Lin X., Chen H. y Xu S. (2013). Spatial distribution and ecotoxicological risk assesment of heavy metals in surface sediments of the Bohai Bay, China. Environ. Sci. Pollut. Res. 20 (6), 4099-4110. DOI: 10.1007/s11356-012-133-Z

Hung J. y Hsu C. (2004). Present state and historical change of trace pollution in Kaoping coastal sediment southwestern, Taiwan. Mar. Poll. Bull. 49 (11-12), 986998. DOI: 10.1016/j.marpolbul.2004.06.028

INE (2014). XIV Censo de Población y Vivienda. Resultados por entidad federal y municipio [en línea]. http:// www.ine.gov.ve/documentos/Demografia/Censo de Población y Vivienda/pdf/sucre.pdf 29/1/2019

Kamau J.N. (2002). Heavy metal distribution and enrichment at Port-Reitz Creek, Mombasa. West. India Ocean. J. Mar. Sci. 1 (1), 65-70.

Krupadam R., Smith P. y Wate R. (2006). Geochemical fractionation of heavy metals in sediments of Tapi estuary. Geoch. J. 40 (5), 513-522.

Ling M.S. y Yang S.X. (2008). Heavy metal contamination in soils and phytoaccumulation in manganese mine wasteland, South China. Air Soil Water Res. I, 31-41. DOI: 10.4137/ASWR.S2041

Lodeiros C. (2002). Una cuestión de peso y de posición. Rev. Biol. Trop. 50 (3-4), 875-878.

Long E.R., MacDonald D.D., Smith S.K.L. y Calder F.D. (1995). Incidence of adverse biological effects within ranges of chemical concentrations in marine and estuarine sediments. Environ. Manage. 19 (1), 81-97. DOI: 10.1007/BF02472006Long E.R., Field L.J. y MacDonald D.D. (1998). Predicting toxicity in marine sediments with numerical sediment quality guidelines. Environ. Toxicol. Chem. 17 (4), 714-727. DOI: $10.1002 /$ etc. 5620170428

Long E.R., Ingersoll C.G. y MacDonald D.D. (2006). Calculation and uses of mean sediment quality guideline quotients: a critical review. Environ. Sci. Technol. 40 (6), 1726- 1736. DOI: 10.1021/es058012d

MacDonald D.R., MacDonald M., O'Brien M. y Gobeil C. (1991). Accumulation of heavy metals ( $\mathrm{Pb}, \mathrm{Zn}$, $\mathrm{Cu}$ ), carbon and nitrogen in sediments from straight of Georgia, B.C., Canada. Mar. Chem. 34 (1-2), 109-135. DOI: 10.1016/0304-4203(91)90017-Q 
MacDonald D.D., Carr R.S., Calder F.D., Long E.R. e Ingersol C.G. (1996). Development and evaluation of sediment quality guidelines for Florida coastal waters. Ecotoxicology 5 (4), 253-278.

DOI: $10.1007 / \mathrm{BF} 00118995$

MacDonald D.D., Ingersoll C.G. y Berger T.A. (2000). Development and evaluation of consensus-based sediment quality guidelines for freshwater ecosystems. Arch. Environ. Contam. Toxicol. 39 (1), 20-31.

DOI: $10.1007 / \mathrm{s} 002440010075$

Martínez G. (2002). Metales pesados en los sedimentos superficiales del Golfo de Cariaco, Venezuela. Bol. Inst. Oceanogr. Venezuela Univ. Oriente 41 (1-2), 83-96.

Martínez G. (2016). Calidad ambiental de los sedimentos recientes del Golfo de Cariaco, Trabajo de Ascenso. Instituto Oceanográfico de Venezuela, Universidad de Oriente, Cumaná, Venezuela, 175 pp.

Márquez A., Bonilla J., Martínez G. y Senior W. (2005). Estudio geoquímico de los sedimentos superficiales del litoral nororiental del Golfo de Cariaco, Estado Sucre, Venezuela. Bol. Inst. Oceanogr. Venezuela Univ. Oriente 44 (2), 89-103.

Márquez A., Senior W., Benítez A., Fermín I., Martínez G., González A., Castañeda J., Alcalá L. y de la Cruz R. (2011). Sector oriental del Golfo de Cariaco, Venezuela. Una descripción dinámica hidroquímica, procesos y rol de la surgencia costera estacional. Bol. Inst. Oceanogr. Venezuela Univ. Oriente 50 (2), 255-272.

Meier P. y Zünd R. (1993). Statical method in analytical chemistry. 2a ed. Wiley Interscience, Nueva York, USA, $320 \mathrm{pp}$.

Montezuma D. (2010). Determinación de análisis de riesgo sísmico, Estado Sucre, Venezuela. Tesis de Maestría. Facultad de Humanidades y Educación, Universidad Central de Venezuela, Caracas, Venezuela, 153pp.

Müller G. (1979). Schwermetalle in den sedimenten des Rheins-Veranderungen seitt. Umschau 79, 778-783.

Nowrousi H.R. y Pourkhabbaz A. (2014). Application of geoaccumulation index and enrichment factor for assesing metal contamination in the sediments of Hara Biosphere Reserve, Iran. Chem. Speciat. Bioavailab. 26 (2), 99-105.

DOI: $10.3184 / 095422914 X 13951584546986$

Ntakirutimana G.D., Guo J., Gao X. y Huang L. (2013). Contamination and potential ecological risk assessment of heavy metals in a lake. Pol. J. Environ. Stud. 22 (4), 1129-1134.

Núñez J.G., Méndez E., Ariza L.A. y Ruiz L. (2011). Variaciones diurnas y nocturnas de la estructura comunitaria de peces en un arrecife de coral frageante en el Golfo de Cariaco, Estado Sucre, Venezuela. Bol. Centro Invest. Biol. 45 (2), 367-386.
Páez-Osuna F. y Osuna-López J. (1992). Diagénesis temprana de metales pesados en sedimentos del Pacífico adyacente a la boca del Golfo de California, México. Geofis. Int. 31 (3), 289-304.

PNUMA (2009). PAC-XX. Golfo de Cariaco (Venezuela). Informe. Programa Mundial para el Medio Ambiente, Kingston, Jamaica, 65 pp.

Ponce R., Forja J. y Gómez A. (2000). Influencia de la actividad antropogénica en la distribución vertical de $\mathrm{Zn}, \mathrm{Cd}, \mathrm{Pb}$ y $\mathrm{Cu}$ en agua intersticial y sedimentos marinos costeros (Bahía de Cádiz, España). Cienc. Mar. 29 (3), 479-502.

Quintero A., Terejova G., Vincent G., Padrón A. y Bonilla J. (2002). Los pescadores del Golfo de Cariaco. Interciencia 27, 286-292.

Quintero A., Terejova G. Bonilla J. (2005). Morfología costera del Golfo de Cariaco, Venezuela. Bol. Inst. Oceanogr. Venezuela Univ. Oriente 44, 133-143.

Quintero A., Caraballo L. F., Bonilla J., Terejova G. y Rivadula R. (2006). Sedimentos marinos costeros del Golfo de Cariaco. Bol. Inst. Oceanogr. Venezuela. Univ. Oriente 45, 127-139.

Reyes Y., Vergara I., Torres O., Díaz M. y González E. (2016). Contaminación con metales pesados: implicaciones en salud, ambiente y seguridad alimentaria. Rev. Ingen. Invest. Desarrollo 16 (2), 66-77.

Rojas L., Chang I., Agard J., Bekele I. y Hubbard R. (2002). Heavy metals in green mussel (Perna viridis) and oysters (Crassostrea sp.) from Trinidad and Venezuela. Arch. Environ. Contam. Toxic. 42 (4), 410-415. DOI: $10.1007 / \mathrm{s} 00244-001-0044-\mathrm{Z}$

Rubio B., Nombela M., Vilas F., Alejo I., García-Gil E. y Pazos O. (1995). Distribución y enriquecimiento de metales pesados en sedimentos actuales de la parte interna de la Ría de Pontevedra. Thalassas 11, 35-45.

Rubio B., Nombela M. y Vilas F. (2000). La contaminación por metales pesados en las Rías Baixas gallegas: nuevos valores de fondo para la Ría de Vigo (NO de España). J. Iber. Geol. 26,121-149.

Salbu B., Krekling T y Outon D.H. (1988). Characterization of radioactive particles in the environment. Analyst. 123, 843-849.

SCFI (1995). Norma Oficial Mexicana NMX-AA-11101995-SCFI 1995. Que establece el método biológico para la evaluación de la calidad de agua mediante pruebas de toxicidad aguda usando Artemia franciscana. Requisitos a que deben ajustarse los terceros que realicen la prueba. Secretaría de Comercio y Fomento Industrial. Diario Oficial de Federación, México, 6 de marzo.

Serveiss V.B. y Ohlson D.W. (2007). Using risk assesment principles in a source water protection assesment. Hum. Ecol. Risk Asses. 13, 402-417. 
Simpson S.L., Batley G.E., Cxhariton A., Astauber J., King C., Chapman J., Hyne R., Gale S., Roach A. y Maher W. (2005). Handbook for sediment quality assesment. CSIRO, Bangor, Nueva Gales del Sur, 126 pp.

Sutherland R.A. (2000). Bed sediment-associated trace metals in an urban stream, Oahu, Hawaii. Environ. Geol. 39 (6), 611-627. DOI: 10.1007/s002540050473

Sung Y.B., Zhou Q.X., Xie X.K. y Liu R. (2010). Spatial sources and risk assesment of heavy metal contamination of urban soils in tipical regions of Shengang. Chin. J. Hazard. Mater. 174 (1-3), 455-462. DOI: 10.1016/j.jhazmat.2009.09.074

Turekian K. y Wedepohl L. (1961). Distribution of the elements in some major units of the earth's crust. Geol. Soc. Am. Bull. 72(2), 175-192. DOI: 10.1130/0016-7606(1961)72[175:doteis]2.0.co;2

Velásquez A. (2005). Distribución y comportamiento de los metales pesados $\mathrm{Cd}, \mathrm{Cu}, \mathrm{Ni}, \mathrm{Zn}, \mathrm{Fe}, \mathrm{Mn}, \mathrm{Co}, \mathrm{Cr}$ y $\mathrm{Pb}$ en los sedimentos superficiales del sector oriental del Golfo de Cariaco, Estado Sucre, Venezuela. Tesis de Licenciatura. Escuela de Ciencias, Universidad de Oriente, Cumaná, Venezuela, 121 pp.
WAC (1995). Norma de gestión de sedimentos del código administrativo de Washington WAC-173-204-320. Calidad estándar de sedimentos marinos. Legislación del Estado de Washington, EUA, 29 de enero de 1996.

Wang S., Jia Y., Wang S., Wang X., Wang H., Zhao Z. y Liu B. (2010). Fractionation of heavy metals in shallow marine sediments from Jinzhou Bay, China. J. Environ. Sci.-China 22 (1), 23-31. DOI: 10.1016/s1001-0742(09)60070-x

Yang J., Cao L., Wang J., Liu C., Huang C., Cai W., Fang H. y Peng X. (2014). Speciation of metals and assesment of contamination in surface sediments from Daya Bay, South China Sea. Sustainability 6 (12), 9096-9113. DOI: 10.3390/su6129096

Zhang J. y Liu C.L. (2002). Riverine composition and estuarine geochemistry of particulate metals in China-Weathering features, anthropogenic impact and chemical fluxes. Estuar. Coast. Shelf Sci. 54(6), 1051-1070. DOI: 10.1006/ecss.2001.0879 